\title{
Study the Influence of Treatment Interruptions in the Radical Irradiation of Breast Cancer
}

\author{
Ahmed H. Oraby ${ }^{1}$, Ibrahim A. Awad ${ }^{2}$, Ehab M. Attalla ${ }^{3}$, and Alia A. Azzam ${ }^{4}$ \\ ${ }^{1}$ Department of Physics, Faculty of Science, Mansoura University, Mansoura, Egypt \\ ${ }^{2}$ Department of Clinical Oncology and Nuclear Medicine, Faculty of Medicine, Mansoura University, \\ Mansoura, Egypt \\ awadonc@gmail.com \\ ${ }^{3}$ Department of Physics, Division of Medical Physics, National Cancer Institute, Cairo University \& \\ Children's Cancer Hospital, Cairo, Egypt \\ attalla.ehab@gmail.com \\ ${ }^{4}$ Department of Clinical Oncology\& Nuclear Medicine, Faculty of Medicine, Mansoura University, \\ Mansoura, Egypt \\ alia_a.azam@yahoo.com
}

\begin{abstract}
:
Purpose: Hypofractionated radiotherapy (HFRT) in breast cancer treatment regimen (40 Grey /15 fractions/3 weeks) is more convenient for patients, especially those coming from remote areas to radiotherapy facilities and for healthcare providers, than conventional fractionation (50 Gy/25 fractions/5weeks). So the effect of radiotherapy interruption on treatment outcome (loco-regional control (LRC)\& overall survival (OS)) during hypofractionated schedule is the issue of our study.

Materials and Methods: We studied retrospectively 174 female patients with breast cancer who received PORT at the Clinical Oncology \& Nuclear Medicine Department, Faculty of Medicine, Mansoura University, Egypt, from January-2012 to December-2016. We determined the treatment outcome (OS\&LRC) from the follow-up (FU) of the studied patients, as the patient still survived or died, and recurrence till now occurred or not, and were estimated with the Kaplan-Meier (KM) method and Logrank test, respectively. Then we calculated surviving fraction (SF) and tumor control probability (TCP) with regard to biologically effective dose (BED), for all patients, using breast cancer radiobiological parameters.

Results: When comparing patients without radiotherapy gap with patients with radiotherapy gaps, the results showed a decrease in LRC rate in patients with radiotherapy treatment interruptions by $15 \%(P=0.019$, a significant value), but no detrimental effect on OS because of the very limited number of the studied patients. Curves of the relationship between (SF\&OTT) and (TCP\&OTT) confirmed the detrimental effect of unscheduled gap during radiotherapy fractions on the treatment outcome. Also we found a significant- $P$ value for (marital status, start day of radiotherapy fractions, time, number, and duration of gaps); it means these factors affect LRC during radiotherapy interruptions.
\end{abstract}

Conclusion: Interruptions during postoperative hypofractionated irradiation of breast cancer (40 Gy/15 fractions/3weeks) should be avoided and if they are inevitable, they should not be prolonged more than two days, as they will adversely affect the treatment outcome (LRC).

Keywords: PORT: Post-Operative Radiotherapy, OTT: Overall Treatment Time, LR: Local Recurrence, HFRT: Hypofractionated radiotherapy, LRC: Loco-Regional Control, OS: Overall Survival, FU: Follow-Up, K-M: Kaplan-Meier, SF: Surviving Fraction, TCP: Tumor Control Probability, BED: Biologically Effective Dose, BCS: Breast Conserving Surgery, NACT: Neoadjuvant Chemotherapy, and PMRT: Postmastectomy Radiotherapy.]

\section{INTRODUCTION}

Worldwide, breast cancer is the most common cancer among females [1]. Treatment of breast cancer includes surgical intervention, radiotherapy (RT), and systemic treatment with cytotoxic chemotherapy (CT), hormone therapy, targeted (biologic) therapy, or a combination of these [2, 3]. Radiation after breast conserving surgery (BCS) for early as well as locally advanced tumor after neoadjuvant chemotherapy (NACT) is now considered as an integral part of Breast Conserving Therapy (BCT) whereas postmastectomy radiotherapy (PMRT) to chest wall and or regional area is considered beneficial for a selected group of high risk patients [4-9]. PMRT decreases loco-regional recurrence (LRR) in women with operable breast cancer and inhances survival [10].

Conventional fractionated radiotherapy has been limited by patient's compliance, travelling, unplanned interruption and others. Hypofractionated schedule would be more appealing and convenient [10]. In the clinical practice, daily, unplanned radiotherapy interruptions are inevitable because of intercurrent disease, acute radiation reactions, machine breakdowns, public holidays, and patient non-compliance [11]. Good clinical Practice (to achieve tumor control) dictates that radical courses of radiotherapy treatment should not be interrupted. Where this is not possible, compensatory treatment is required [12].

Recently it had been shown that radiotherapy interruptions lasting more than a week decreased LRC and OS (patient outcomes) rates in breast cancer patients treated with postoperative radiotherapy (PORT) after breast-conserving surgery 
and mastectomy $[11,13]$. But there are no reports for the effect of unscheduled treatment gaps in the radical irradiation of breast cancer on patient outcomes, in case of hypofractionation regime (40 Gy per 15 fractions over 3 weeks), thus it will be our issue in this study using retrospective analysis.

\section{PATIENTS\&METHODS}

\subsection{Patients}

174 female breast cancer patients who received postoperative radiotherapy (in the year 2012 and 2013 inclusive), at the Clinical Oncology \& Nuclear Medicine Department, Faculty of Medicine, Mansoura University, Egypt, were retrospectively analyzed.

All the studied patients underwent surgery as the primary treatment modality of breast cancer; 21 patients (12.1\%) underwent breast-conservative surgery while 153 patients (87.9\%) underwent mastectomy.

\subsection{Methods}

\subsubsection{Radiotherapy Fractions}

Irradiation of chest wall or breast was with two tangential medial and lateral beams. irradiation of Lymph nodes (supraclavicular, axillary and internal mammary chain) was with an anterior one direct beam at $3 \mathrm{~cm}$ depth, or two parallel opposed fields (dose to axilla was supplemented with a posterior beam). Patients were irradiated with the linear accelerator unit; by $6 \mathrm{MV}$ or $15 \mathrm{MV}$ of the ELEKTA; Precise, $6 \mathrm{MV}$ of the SL75/5 photon beams, or $6,8,10,12$, and 15 electron beams from the ELEKTA; Precise. The choice of the machine and energy type is achieved according to patient weight, and (the target volume and depth), respectively. A total dose of 40 Gy (hypofractionated radiation therapy (HFRT)) was delivered to the breast or the chest wall and regional lymph nodes in 2. 67 Gy per fraction (5 daily fractions in a week) from Saturday to Wednesday; conventional fractionation, according to the treatment tactic of the department. The overall treatment time (OTT) was 19 days if the patient started radiotherapy fractions on Saturday, or 21 days if patient started on any other day from Sunday to Wednesday. A 16 Gy (2 Gy daily fraction), or 9 Gy (3Gy weekly fraction) boost dose (electron or photon beams) was delivered to tumor bed after breast-conservative surgery.

\subsubsection{Adjuvant Systemic Therapy}

chemotherapy or hormonal therapy was given to 174 patients $(100 \%)$. One hundred and twenty-two patients $(69.7 \%)$ received hormonal treatment (tamoxifen). One hundred and seventy-two patients (98.8\%) received chemotherapy, and 120 patients (68.9\%) received chemotherapy and hormonal therapy. Some patients received chemotherapy following postoperative irradiation; however, the others finished chemotherapy before irradiation or received a few cycles before irradiation (a sandwich regimen). Chemotherapy regimen consisted of: (a) 6 cycles of CMF (cyclophosphomide; methotraxate; 5-fluouracil). (b) Or 6 cycles of CAF or CEF (doxorubicin (A) or epirubicin (E)); an anthracycline based regimen. (c) Or 4 cycles of AC or EC (A 60 or E 90). Thirty five patients (20.1\%) with HER2- positive breast cancers received targeted therapy (Trastuzumab or Herceptin). Ninety three patients (53.45\%) were HER2negative breast cancers, and fourty six cases (26.4\%) were unknown.

\subsubsection{Determination of Radiotherapy Interruptions}

Weekends (Thursday and Friday) as they are integral parts of the conventional radiotherapy scheme, Thursdays and Fridays were not considered as radiotherapy breaks; however, if the gap was before or after a weekend, Thursday and Friday were included in the interruption. In calculation, the total number of days of interruption was summed and processed as weeks. Unscheduled radiotherapy breaks occurred in 129 patients (74.1\%). Among these 129 patients the mean and median durations of the gaps were 17 and 13 days, respectively, and range was (1-89 days). For all the studied patients (174 patients), including 45 patients without radiotherapy interruption, the mean and median duration of the interruptions were 16 and 12 days, respectively. When gaps number was estimated, 51 patients (29.3\%) had one radiotherapy gap, while 84 patients $(48.3 \%)$ had more than one treatment gap. In 22 patients (17\%) early skin reaction was the main cause of interruption.

According to the duration of treatment interruptions all the studied patients were distributed into 4 groups. Group (A) include 45 patients $(25.9 \%)$ who had no radiotherapy break. Group (B) include 87 patients $(50 \%)$ with radiotherapy interruptions $\leq 7$ days ( $\leq 1$ week). Group (C) include 26 patients $(14.9 \%$ ) with radiotherapy gaps $\geq 8$ days and $\leq 14$ days. Group (D) include 16 patients $(9.2 \%)$ with radiotherapy gaps $\geq 15$ days or more.

\subsubsection{Radiobiological Calculations}

To calculate surviving fraction (SF) and tumor control probability (TCP) with regard to biologically effective dose (BED), by using breast cancer radiobiological parameters and the following equations:

$B E D=n . d[1+d \div(\alpha / \beta)]-K(T-T d e l a y) \rightarrow$ Eq. $(1)[12,14-16]$

$\ln (S F)=-E[17,18]$ 
dividing by $\alpha$ :

$\ln (S F) / \alpha=-E / \alpha=-B E D \ln (S F)=-\alpha . B E D$

then,

$S F=[\exp .(-\alpha . B E D)] \rightarrow E q .(2) \mathrm{TCP}=[\exp .(-N 0 . S F)]$

$\mathrm{TCP}=\{$ exp. [-N0. exp. (-a. BED)] $\rightarrow$ Eq. (3) [15]

Where: $\mathbf{n}$ (the number of well-spaced and identical fractions) $=15$ fractions, $\mathbf{d}$ (the dose per fraction) $=2.67$ Gy (hypofractionation regimen $40 \mathrm{~Gy} / 15$ fractions) [16]. ( $\alpha / \beta)$ ratio (is the linear to quadratic coefficients in the linear quadratic (LQ) model, and represents the dose at which single and double-hit killing events are equal in number; at higher doses double-hit killing events start to progressively dominate) = 4 Gy [19].

K (tumor repopulation factor) $=\ln 2 / \alpha . \mathbf{T d}=0.693 / \alpha$. Td (potential doubling time), $\mathrm{Td}=13$ days, $\alpha=0.3 \mathrm{~Gy}-1[20,16]$.

$\mathbf{T}$ (overall treatment time) $=19$ days or 21 days if the patient started radiotherapy fractions on Saturday or midweek respectively [16]

Tdelay (the days between the start of radiotherapy and the onset of tumor repopulation) = 14 days [16].

NO (the initial number of clonogens in the tumor) $=109$. V [15]. The density of clonogens per cubic centimeter (cc) $=109$ [15]. There is general agreement in the literature that $1 \mathrm{~cm} 3$ tumor mass contains, $10^{9}$ cells [21]. We considered $\mathrm{V}$ (actual volume of the tumor) $=10-2 \mathrm{cc}$ after surgery for all patients in our study.

\subsubsection{Patients' Data}

Collect host and treatment-related factors: age, weight, height, marital status, menopausal status, histological types of the tumor, grade of the tumor, PT stage and PN stage of the tumor according to the clinical TNM Staging System, start day of radiotherapy, time, number, and duration of gaps, and treatment outcomes (overall survival (OS) and loco-regional control (LRC)) from the follow-up (FU) of the patient, as the patient still survived or died, and recurrence till now occurred or not.

\subsubsection{Statistical Analysis}

We used SPSS Statistical package for social science. The qualitative data were presented in the form of number and percentage. Chi-square used as a test of significance. The quantitative data were presented in the form of mean and standard deviation. Student t test was used for Quantitative data. Correlation was used to study the correlation between data. KaplanMeier [22] Survival analysis was done to estimate the probability of survival. Significance was considered at $p$ value less than 0.05 .

\section{RESULTS\&DISCUSSION}

\subsection{Results}

The LRC rate of 45 patients with no radiotherapy gap (group A) for 5 years was (93.3\%), and the LRC rate of 129 patients with radiotherapy gaps (groups B,C +D) was $(78.3 \%)$ for 5 years. When we compared the patients with radiotherapy gaps with the patients without radiotherapy gap, the difference was statistically significant $(p=0.019)$ by LOGRANK test; figure (1) and table (1).

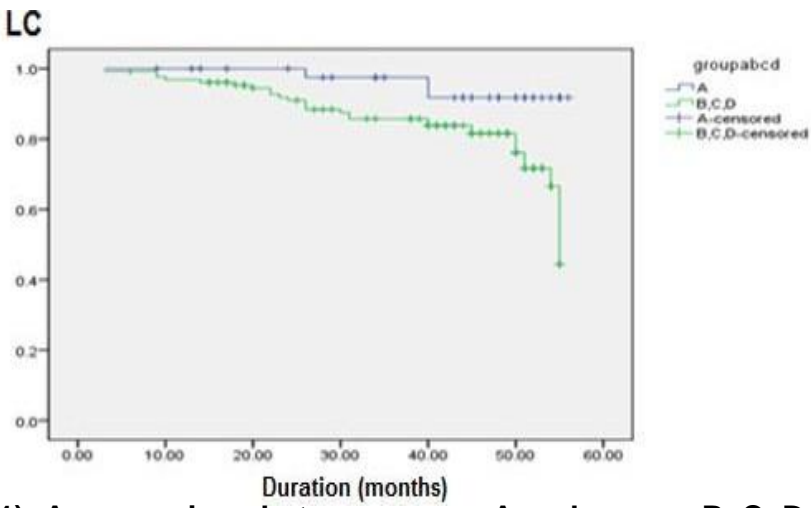

Figure (1): A comparison between group $A$ and groups $B, C+D$ for $L R C$.

We could not observe a detrimental effect of interruptions on $O S$ rates $(p=0.81)$, or the difference between group $A$ and groups $B, C+D$ was not statistically significant, and this was due to the very poor number of patients in each group, and estimations in larger patient groups would further clarify the hypothesis of the negative effect of decreased LRC on OS; table (1). But it has been proven theoretically the bad effect of interruption on both tumor control probability (TCP) and surviving fraction (SF); figures (2) and (3). 
Table (1): Comparison of treatment outcome between group A and Groups B, C+D.

\begin{tabular}{|c|c|c|c|}
\hline Patient & $\begin{array}{l}\text { Group (A) } \\
\text { Patients in } \\
\text { No. (\%) }\end{array}$ & $\begin{array}{l}\text { Groups }(B+C+D) \\
\text { Patients in No. (\%) }\end{array}$ & $\begin{array}{l}\text { Test of } \\
\text { Significance }\end{array}$ \\
\hline $\begin{array}{l}\text { Recurrence: } \\
\text { No }\end{array}$ & 42 (93.3) & 101 (78.3) & $0.019^{* *}$ \\
\hline $\begin{array}{r}\text { Survival: } \\
\text { Dead Survived }\end{array}$ & $7(15.6)$ & $22(17.1)$ & 0.81 \\
\hline
\end{tabular}

The relationship between tumor control probability (TCP)\&overall treatment time (OTT) in days, and between surviving fraction (SF)\&overall treatment time (OTT) in days, in the comparison between group $A$ and groups $B, C+D$ are shown in figures (2) and (3), respectively by correlation. In figure (2), the slope of the curve expressed in groups $B, C+D$ increased down after 19 days (the overall treatment time with not-interrupted radiotherapy fractions or compliant radiotherapy). The curve expressed in groups B, C+D shows a decreased TCP (measured in \%) with increased OTT in days, and the curve expressed in group A shows the higher TCP (measured in \%) with the overall treatment time 19 days (the compliant radiotherapy course).

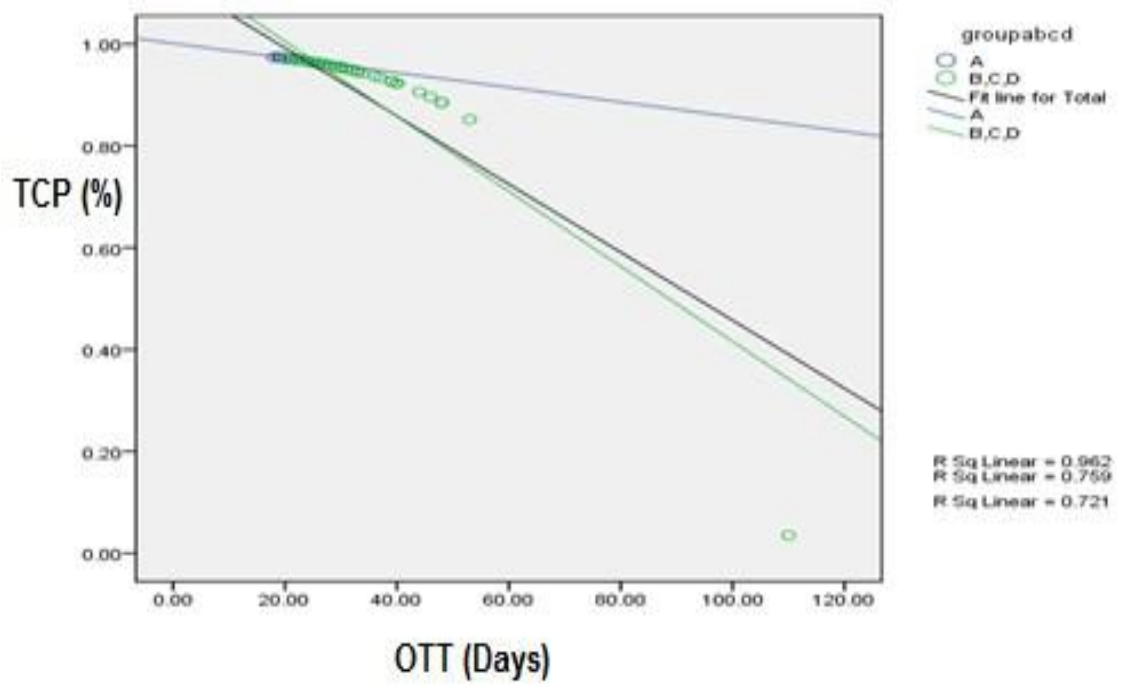

Figure (2): Relationship between OTT \& TCP.

In figure (3), the slope of the curve expressed in groups B, C+D increased up after 19 days (compliant radiotherapy course). The curve expressed in groups B, C+D shows an increased SF (measured in number of surviving cells) with increased OTT (in days), and the curve expressed in group A shows the lower SF (measured in number of surviving cells) with OTT= 19 days (the compliant radiotherapy course). 


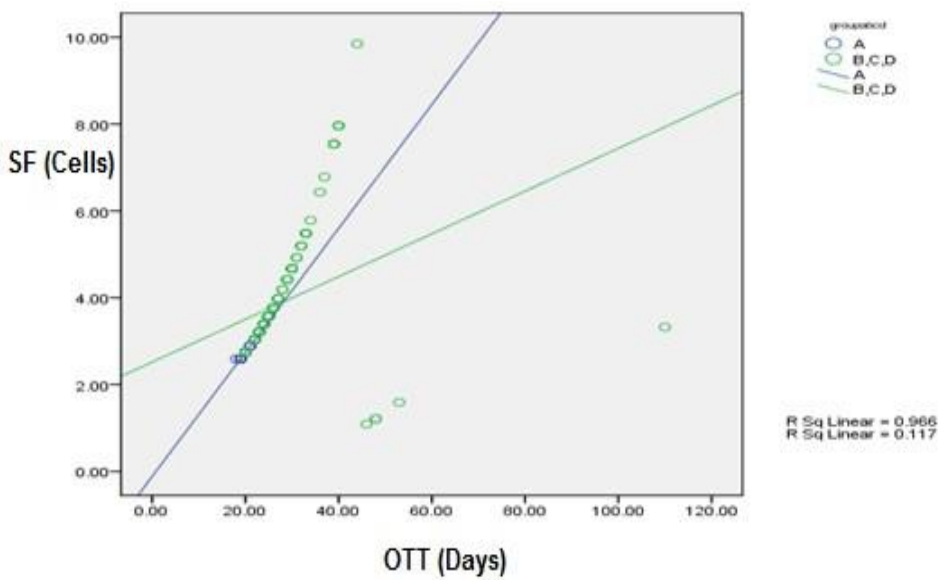

Figure (3): Relationship between OTT \& SF.

When we compared demographic characteristics of the patients without radiotherapy interruption with the patients with radiotherapy breaks, it was found that the distribution of the patients according to marital status was differentially highly significant $(p=0.001)$, but it was non- significant for age $(p=0.66)$, weight $(p=0.37)$, height $(p=0.14)$, menopausal status $(p=0.92)$, by Chi-square test as a test of significance; table (2).

Table (2): Influence of some demographic factors on LRC in the comparison between group A and groups B, C+D.

\begin{tabular}{|c|c|c|c|}
\hline Character & $\begin{array}{l}\text { Group (A) } \\
\text { Patients in } \\
\text { No.(\%) }\end{array}$ & $\begin{array}{l}\text { Groups } \\
(B+C+D) \\
\text { Patients in }\end{array}$ & $\begin{array}{l}\text { Test of } \\
\text { Significance }\end{array}$ \\
\hline $\begin{array}{l}\text { Age: } \\
\leq 49 \text { years }\end{array}$ & $21(46.7)$ & $65(50.4)$ & 0.66 \\
\hline $\begin{array}{l}\text { Marital Status: } \\
\text { Missing } \\
\text { D M S W }\end{array}$ & $\begin{array}{l}5(11.1) \\
1(2.2) \\
35(77.8)\end{array}$ & $\begin{array}{l}18(13.95) \\
2(1.55) \\
83(64.3)\end{array}$ & $0.001^{* * *}$ \\
\hline $\begin{array}{l}\text { Weight: } \\
\leq 89 \text { kgs } \\
>89 \text { kgs Missing }\end{array}$ & $\begin{array}{l}22(48.9) \\
15(33.3)\end{array}$ & $\begin{array}{l}54(41.9) \\
58(45)\end{array}$ & 0.37 \\
\hline $\begin{array}{l}\text { Height: } \\
\leq 158 \mathrm{~cm} \\
>158 \mathrm{~cm} \text { Missing }\end{array}$ & $\begin{array}{l}27(60) \\
9(20) \\
\end{array}$ & $\begin{array}{l}64(49.6) \\
46(35.7) \\
\end{array}$ & 0.14 \\
\hline $\begin{array}{l}\text { Menopausal } \\
\text { Status: } \\
\text { Premenopausal } \\
\text { Postmenopausal }\end{array}$ & $23(51.1)$ & 67 (51.9) & 0.92 \\
\hline
\end{tabular}

Chi-square test showed that grade, histological type, T stage, and $\mathrm{N}$ stage had not significantly influenced LRC $(\mathrm{p}=0.81, p=0.60, p=0.42$, and $\mathrm{p}=0.32$ respectively); table (3). 
Table (3): Influence of histological and pathological factors on LRC in the comparison between group A and groups $\mathrm{B}, \mathrm{C}+\mathrm{D}$.

\begin{tabular}{|c|c|c|c|}
\hline Character & $\begin{array}{l}\text { Group (A) } \\
\text { Patients in } \\
\text { No. (\%) }\end{array}$ & $\begin{array}{l}\text { Groups } \\
(B+C+D) \\
\text { Patients in No. }\end{array}$ & $\begin{array}{l}\text { Test of } \\
\text { Significance }\end{array}$ \\
\hline \multicolumn{4}{|c|}{$\begin{array}{l}\text { Histological Type: } \\
\text { Ductal }\end{array}$} \\
\hline Non-ductal & $39(86.7)$ & $110(85.3)$ & 0.81 \\
\hline \multicolumn{4}{|l|}{ Grade: } \\
\hline G1 & $1(2.2)$ & $3(2.32)$ & 0.60 \\
\hline G2 & $26(57.8)$ & 88 (68.22) & \\
\hline $\mathrm{G} 3$ & $10(222)$ & $23(1783)$ & \\
\hline \multicolumn{4}{|l|}{ T stage: T1 } \\
\hline T2 T3 T4 & $3(6.7)$ & $15(11.6)$ & 0.42 \\
\hline \multirow[t]{2}{*}{ Tx (unknown) } & 28 (62.22) & $62(48.1)$ & \\
\hline & $11(24.44)$ & $33(25.6)$ & \\
\hline \multicolumn{4}{|l|}{ N stage: N0 } \\
\hline N1 N2 N3 & $7(15.5)$ & $26(20.2)$ & 0.32 \\
\hline \multirow[t]{2}{*}{ Nx (unknown) } & $13(28.9)$ & 37 (28.7) & \\
\hline & $13(28.9)$ & $33(25.6)$ & \\
\hline
\end{tabular}

Chi-square test showed that in all the Start day of RT fractions, number of gaps, time of gap, and duration of gap, the difference was statistically highly significant $(p=0.001)$, and independently related with LRC; table (4).

Table (4): Influence of radiotherapy fractions on LRC in the comparison between group $A$ and groups $B, C+D$.

\begin{tabular}{llll}
\hline Character & $\begin{array}{l}\text { Group (A) } \\
\text { Patients in } \\
\text { No. (\%) }\end{array}$ & $\begin{array}{l}\text { Groups }(\mathrm{B}+\mathrm{C}+\mathrm{D}) \\
(\%)\end{array}$ & $\begin{array}{l}\text { Test } \\
\text { Significance }\end{array}$ \\
\hline $\begin{array}{l}\text { Start Day of } \\
\text { Fractions: }\end{array}$ & & & \\
Saturday & & & \\
Sunday & $28(62.22)$ & $71(55.04)$ & $0.001^{* * *}$ \\
Monday & $11(24.44)$ & $22(17.05)$ & \\
$\begin{array}{l}\text { Tuesday } \\
\text { Wednesday }\end{array}$ & $4(8.9)$ & $16(12.4)$ & \\
& $2(4.44)$ & $15(11.6)$ & \\
\hline
\end{tabular}




\begin{tabular}{llll}
\hline No. of Gaps: & & \\
0 & $39(86.7)$ & $0(0)$ & $0.001^{* \star *}$ \\
1 & $6(13.3)$ & $44(34)$ & \\
2 & $0(0)$ & $45(34.9)$ & \\
3 & $0(0)$ & $34(26.4)$ & \\
4 & $0(0)$ & $5(3.9)$ & \\
5 & $0(0)$ & $1(0.8)$ & \\
\hline
\end{tabular}

Timing of gaps:

\begin{tabular}{llll}
0 & $39(86.7)$ & $0(0)$ & $0.001^{* \star *}$ \\
first & $1(2.2)$ & $3(2.32)$ & \\
second & $1(2.2)$ & $26(20.2)$ & \\
third & $4(8.9)$ & $22(17.05)$ & $13(10.08)$ \\
first, second & $0(0)$ & $10(7.75)$ & \\
first, third & $0(0)$ & $27(20.9)$ & \\
second, third & $0(0)$ & $28(21.7)$ & \\
first, second, third & $0(0)$ & \\
\hline Length (duration) & & \\
of interruption or & & \\
prolonged days: & & $31(24.03)$ \\
0 & $45(100)$ & $8(6.2)$ \\
1 & $0(0)$ & $48(37.21)$ \\
2 & $0(0)$ & $26(20.16)$ \\
$(3-7)$ & $0(0)$ & $16(12.4)$ \\
$(8-14)$ & $0(0)$ & $0(0)$ & \\
$>14$ & &
\end{tabular}

\subsection{Discussion}

Bese N. S. et al. had the first clinical paper evaluated the impact of radiotherapy breaks in postoperative radiotherapy of breast cancer during the schedule (50 Gy/25 fractions/5 weeks). They found when the duration of radiotherapy interruption was prolonged over 7 days, a negative effect was found on both OS and LRC, and the average decrease in the LRC rate was $5 \%$ along 5-years, but there were no detrimental impact on patient treatment outcome if the duration of gap lasted for period $\leq 1$ week [11].

Bese N. S. et al. repeated the previous study on ostoperative irradiation interruptions after breast- onserving Surgery. They also found that prolongation of more than one week negatively impact LRC by a $9 \%$ ecrease in the 5 -year LRC rate [13].

Nitin Ohri et al. reviewed all patients who completed ourses of external beam RT with curative intent in their epartment from the years 2007 to 2012 for cancers of the ead and neck, breast, lung, cervix, uterus, or rectum. atients who missed $\geq 2$ days (excluding planned eatment breaks) were deemed noncompliant. They ported that RT noncompliance was associated with creased recurrence risk, 5 -year cumulative incidence $16 \%$ ersus $7 \%, P<0.001$ [23].

We have done the first clinical and physical study estimating the impact of radiotherapy gaps in postoperative radiotherapy of breast cancer in the schedule (40 Gy/15 fractions/3 weeks). In our work, we found that no detrimental impact on the treatment outcome of the patient with duration of gap $\leq 2$ days (as the patients with duration of interruption $\leq 2$ days represents $30 \%$ only in groups B; table (4), in addition to those patients are included in group B which have the big number of samples $87(50 \%)$ of all the studied patients (all groups A, B, C, and D), which is the major point of weakness in our study (sample size is very limited) or the population number is very heterogeneous as it is a retrospective study, and the influence of unscheduled interruptions can be shown only if data is analyzed retrospectively. 
Figure (1) describes a comparison between group $A$ and groups $B, C+D$ for $L R C$, in which the green curve represents groups $B, C+D$ curved down and more steeper gradually with time. The blue line is straight but at three points because of the three recurrence cases in the group.

Figure (2) shows the relationship between OTT \& calculated TCP, in which the beginning point of the blue line (group A) appeared at 19 days, the time of compliant radiotherapy course, the higher point on the green line (groups $B, C+D)$ appeared after that and is steeper gradually down to (4\%). The mean value of $\mathrm{TCP}=96 \%$ and $95 \%$ in groups A and B, respectively.

Figure (3) shows the relationship between OTT \& SF, in which also the beginning point of the blue line (group A) appeared at 19 days, the time of compliant radiotherapy course, points on the green line (groups $B, C+D)$ appeared after that and is steeper gradually up to 9.85 cells; which is the meaning of surviving cells. The mean value of $S F=3.49$ and 3.51 in groups $A$ and $B$, respectively.

From table (2) the marital status had influenced $L R C$, but the rest factors did not have any influence on LRC. A trial to study separately the effect of marital status on LRC and by the radiotherapy interruption, the size of patients, sample is small and not enough to estimate the small difference at a significant or non-significant value; table (2). In Bese N. S. et al., the distribution of the patients according to menopausal status was differentially significant $(p=0.04)$ [11].

From table (3) histological\& pathological factors had not significantly influenced treatment outcome. Bese N. S. et al. found that there were no significant differences for the distributions of $N$ stage $(P=0.555)$, T stage $(p=0.984)$, and the adjuvant systemic treatment $(\mathrm{p}=0.172)$. Distribution of patients according to type of surgery when compared with the Pearson_ 2 test, was significantly different $(p=0.005)$ between the two groups [11].

In table (4) the difference was statistically highly significant, and independently related with LRC. This significant value leads us to do separate studies to analyze the effect of each factor alone. Major number of the patients in group A (patients without radiotherapy interruptions) started RT at the beginning of the week; 28 patients (62.2\%), while a higher percentage of patients in groups with interruption started RT mid-week; 58 patients $(45 \%)(p=0.001)$. If the patients start radiotherapy fractions on Saturday, the overall treatment time (OTT) will be 19 days, but if they started on mid-week, the overall treatment time (OTT) will be 21 days by adding weekends. It means that starting radiotherapy midweek prolonged OTT, and affected LRC. There were detected gaps in group A, 6 patients (13.3\%) by a one day, but these gaps had been compensated during fractions, either by on weekend or twice fractions in one day with 6 hours apart, thus resulted in no prolongation in OTT.

In our study, in 22 patients (17\%) the reason for interruption was skin reaction early, and in 2 patients (1.6\%) treatment delay was due to reasons related to medical status of the patients. The other reasons of radiotherapy breaks were public holidays, servicing of the machine, machine breakdowns and noncompliance of patient in 105 patients (81.4\%). The position (time) of the gaps was analyzed, and it was found that the distribution of the patients according to time of gap was differentially highly significant $(p=0.001)$, although in 85 patients $(66 \%)$ the number of radiotherapy interruptions was more than one.

Bese N. S. et al. reported that the reason for gap in $55 \%$ of the patients was early skin reaction and radiotherapy treatment delay was due to leukopenia in $9 \%$ of the patients. There were no other reasons for the gaps related to medical status of the patients were observed. Other causes of gaps were public holidays, breakdowns of machine, servicing of machine and noncompliance of patient. The time of the breaks was not analyzed because the number of radiotherapy interruptions was more than one in $68 \%$ of the patients [11].

It is impossible to prevent some radiotherapy breaks such as those resulting from a bad clinical response to treatment or machine breakdowns, but those resulting from the public holidays can be preplanned. We have to make great efforts to reach the optimized radiotherapy treatment plan to avoid increased doses on skin. Awareness of staff involved in the treatment and patients about the rationale and the importance of the radiotherapy gaps is a necessity. There are guidelines for compensating for interrupted radiotherapy, the Royal College of Radiologists (RCR), in 2008, published the third edition of "Guidelines for the Management of the Unscheduled Interruption or Prolongation of a Radical Course of Radiotherapy" to help departments of clinical oncology in producing local protocols for compensation [12].

\section{CONCLUSION}

We studied the patients retrospectively in this paper, and we found that in the postoperative radiotherapy of breast cancer, gaps for more than two days during radiotherapy schedule (40 Gy/15 fractions/3 weeks) had a detrimental effect on treatment outcome in term of LRC.

\section{ACKNOWLEDGMENTS}

The authors are grateful to all workers in the Clinical Oncology \& Nuclear Medicine Department, Faculty of Medicine, Mansoura University, for their kind help and support. 


\section{REFERENCES}

[1] Amr Abd El-Aziz Ghannam and Rasha Abd El-Ghany Khedr, (2016), "An accelerated hypofractionated schedule with a daily concomitant boost after breast conservation surgery: the feasibility and toxicity." Journal of the Egyptian National Cancer Institute; 28: [39-44].

[2] Carlson R. W., Allred D. C., Anderson B. O., Burstein H. J., Edge S. B., Farrar W. B., Forero A., Giordano S. H., Goldstein L. J., Gradishar W. J., Hayes D. F., Hudis C. A., Isakoff S. J., Ljung B. M. E., Mankoff D. A., Marcom P. K., Mayer I. A., McCormick B., Pierce L. J., Reed E. C., Smith M. L., Soliman H., Somlo G., Theriault R. L., Ward J. H., Wolff A. C., Zellars R., Kumar R., and Shead D. A., (2012), "Metastatic breast cancer, version 1.2012: featured updates to the NCCN guidelines." Journal of National Comprehensive Cancer Network; 10: [821-829].

[3] Chen Hui Tan, Ting-Ting Chao, Jui-Chen Liu, Chun-Hung Lin, Yung-Sung Huang, Chung-Ming Chang, Hon-Yi Lin, YuChieh Su, Yen-Lin Chen, and Ching-Chih Lee, (2016), "Breast cancer therapy and age difference in cardiovascular disease risks: A population-based cohort study in Taiwan." Taiwanese Journal of Obstetrics \& Gynecology; 55: [98-103].

[4] Fisher B., Anderson S., Bryant J., Margolese R. G., Deutsch M., Fisher E. R., Jeong J.-H., and Wolmark N., (2002), "Twenty-year follow-up of a randomized trial comparing total mastectomy, lumpectomy, and lumpectomy plus irradiation for the treatment of invasive breast cancer." The New England journal of medicine; 347: [1233-1241].

[5] Veronesi U., Cascinelli N., Mariani L., Greco M., Saccozzi R., Luini A., Aguilar M., and Marubini E., (2002), "Twentyyear follow-up of a randomized study comparing breast- conserving surgery with radical mastectomy for early breast cancer." The New England Journal of Medicine; 347: [1227-1232].

[6] Overgaard M., Hansen P. S., Overgaard J., Rose C., Andersson M., Bach F., Kjaer M., Gadeberg C. C., Mouridsen H. T., Jensen M. B., and Zedeler K., (1997), "Postoperative radiotherapy in high-risk premenopausal women with breast cancer who receive adjuvant chemotherapy." Danish Breast Cancer Cooperative Group 82b Trial. The New England Journal of Medicine; 337: [949-955].

[7] Overgaard M., Jensen M. B., Overgaard J., Hansen P. S., Rose C., Andersson M., Kamby C., Kjaer M., Gadeberg C. C., Rasmussen B. B., Blichert-Toft M., and Mouridsen H. T., (1999), "Postoperative radiotherapy in high-risk postmenopausal breast-cancer patients given adjuvant tamoxifen: Danish Breast Cancer Cooperative Group DBCG 82c Trial." Lancet; 353: [1641-1648].

[8] Poortmans P., (2007), "Evidence based radiation oncology: breast cancer." Radiotherapy and Oncology; 84: [84-101].

[9] Mondal D. and Sharma D. N., (2016), "External beam radiation techniques for breast cancer in the new millennium: New challenging perspectives." Journal of the Egyptian National Cancer Institute; 28: [211-218].

[10] Elsayed M. Ali and Magdy Khalil Abd AlMageed, (2014), "Post-mastectomy Hypofractionation Radiotherapy in Breast Cancer Patients." Cancer and Oncology Research; 2: [87-93].

[11] Bese N. S., Sut P. A., and Ober A., (2005), "The effect of treatment interruptions in the postoperative irradiation of breast cancer." Oncology; 69: [214-223].

[12] Royal College of Radiologists (RCR), (2008), "The timely delivery of radical radiotherapy: standards and guidelines for the management of unscheduled treatment interruptions. 3rdedition." London: The Board of Faculty of Clinical Oncology.

[13] Bese N. S., Sut P. A., Sut N., and Ober A., (2007), "The impact of treatment interruptions on locoregional control during postoperative breast irradiation." Journal of BUON; 12: [353-359].

[14] Fowler J. F., (2010), "21 years of Biologically Effective Dose." The British Journal of Radiology; 83: [554-568].

[15] Hedman M., Björk-Eriksson T., Brodin O., and Toma-Dasu I., (2013), "Predictive value of modelled tumour control probability based on individual measurements of in vitro radiosensitivity and potential doubling time." The British Journal of Radiology; 86: [20130015].

[16] Douglas W. Arthur, Frank A. Vicini, David E. Wazer, and Atif J. Khan, (2016), "Short Course Breast Radiotherapy. A Comprehensive Review of Hypofractionation, Partial Breast, and Intra-Operative Irradiation." Springer International Publishing Switzerland.

[17] Fowler J. F., (1989), "A Review: The linear quadratic formula and progress in fractionated radiotherapy." The British Journal of Radiology; 62: [679-694].

[18] Paul Jacobs, Aaron Nelson, and lan Liu, (2012), "Biological Effective Dose and Tumor Control Probability Modeling using the MIM® Software Suite." WHITE PAPER, MIM Software Inc., 25200 Chagrin Blvd. Cleveland, Ohio 44122• $866-$ 421-2536 • www.mim software.com

[19] Hennequin C. and Dubray B., (2013), "Le rapport alpha/ bêta revisité à I'heure de l'hypofractionnement." Cancer/ Radiothérapie; 17: [344-348].

[20] Cancer Group Institute, (2011), "Currently Recruiting Breast Cancer Clinical Trials." www.cancergroup.com

[21] Xinfeng Liu, Sara Johnson, Shou Liu, Deepak Kanojia, Wei Yue, Udai P. Singh, Qian Wang, Qi Wang, Qing Nie, and 
Hexin Chen, (2013), "Nonlinear Growth Kinetics of Breast Cancer Stem Cells: Implications for Cancer Stem Cell Targeted Therapy." SCIENTIFIC REPORTS; 3: [2473]. DOI:10.1038/srep02473,http://www.nature.Com/scientificreports.

[22] Kaplan E. L. and Meier P., (1958), "Nonparametric estimation from incomplete observations." J Am Stat Soc B; 53: [457-481].

[23] Nitin Ohri, Bruce D. Rapkin, Chandan Guha, Shalom Kalnicki, and Madhur Garg, (2016), "Radiation Therapy Noncompliance and Clinical Outcomes in an Urban Academic Cancer Center." International Journal of Radiation Oncology ${ }^{\star}$ BiologyPhysics; 95: [563-570]. 